XIAOJUN YU, Ph.D. Student

E-mail: xjyu-myu@163.com

1. School of Economics and Management

Beijing University of Aeronautics and Astronautics

Beijing 100191, China

2. School of Mathematics and Statistics

Guizhou College of Finance and Economics

Guiyang 550004, China

HAI-JUN HUANG, Ph.D.

E-mail: haijunhuang@buaa.edu.cn

School of Economics and Management

Beijing University of Aeronautics and Astronautics

Beijing 100191, China
Science in Traffic and Transport Original Scientific Paper Accepted: Nov. 23, 2009 Approved: Sept. 15, 2010

\title{
EFFICIENCY LOSS OF MIXED EQUILIBRIUM BEHAVIORS WITH POLYNOMIAL COST FUNCTIONS
}

\begin{abstract}
This paper investigates the efficiency loss of selfish routing simultaneously with user equilibrium (UE) player and Cournot-Nash (CN) players. The upper bound of the efficiency loss of UE-CN mixed equilibrium with polynomial cost functions is obtained by the scaling method and the nonlinear programming method, respectively. It is shown that the upper bound of efficiency loss obtained by the scaling method only depends on the polynomial degree of the link travel cost function and the upper bound of efficiency loss obtained by the nonlinear programming method depends on the number of the $\mathrm{CN}$ players besides the aforementioned factors. The numerical tests validate our analytical results.
\end{abstract}

\section{KEY WORDS}

efficiency loss, UE-CN mixed equilibrium, variational inequality, selfish routing game

\section{INTRODUCTION}

Concerning the routing choice behaviour in networks, Wardrop presented two famous principles: the user equilibrium (UE) and the system optimum (SO) ${ }^{1}$. The UE principle states that every traveller aims to minimize their own cost so that no traveller can reduce the cost by unilaterally changing their choice at the equilibrium. In the game theory version, the traffic assignment under the UE principle can be regarded as a non-cooperative Nash game with many infinitesimal users, and all users are competitive to each other. It is, in the game terminology, a non-atomic routing game. In the case of SO, it is assumed that there exists a central organization to minimize the total cost of the system, all users being cooperative and instructed by a single player. The above two principles only consider the two extreme cases, i.e., there are many infinitesimal players in the UE principle and there is only one central player in the SO principle. Haurie and Marcotte investigated the network with some non-cooperative Cournot-Nash (CN) players, where the users belonging to the same player can fully cooperate with each other and different players will compete with each other ${ }^{2}$. The users of one CN player aim to minimize their own total cost while competing with the users of other players. Harker examined that the network users can be divided into different CN players and UE users and obtained a new network equilibrium model ${ }^{3}$. Yang et al. considered such a situation in which the network has an additional player aiming to improve the overall system performance by controlling part of flows and further extended the above model ${ }^{4}$. Recently, Yang and Zhang studied the existence of anonymous link tolls in network with UE-CN mixed equilibrium behaviours ${ }^{5}$

It is well known that the UE is generally not identical with the SO as viewed from the total system cost. However, the gap between the SO and the UE in the worst case had been unknown for a long time. Papadimitriou presented the efficiency loss (the price of anarchy) to measure the efficiency loss for the user's selfish behaviour and defined it as the largest ratio between the total cost of Nash equilibrium and the total cost of an optimal solution achieved by centralized control $^{6}$. Later, Roughgarden and Tardos introduced it into the traffic network and used it to quantify the difference between the UE and the $\mathrm{SO}^{7}$. Recently, researchers have extended the above works in different aspects $^{8-10}$. However, these researches are restricted to the non-atomic routing game, i.e. the routing game only has the UE player with homogeneous users. In addition to non-atomic routing games, Cominetti et al. 
studied the efficiency loss in the atomic games with splittable flows ${ }^{11}$. Yang et al. investigated the efficiency loss of the selfish routing with the atomic $\mathrm{CN}$ players ${ }^{12}$.

However, few scholars studied the efficiency loss of the network simultaneously with UE player and CN players. In this paper, we study the efficiency loss of UE-CN mixed equilibrium. We suppose that all users following the UE principle in their routing decisions are considered as one single UE player and every CN player controls a strictly positive splittable flow. Under this assumption, we use two methods to investigate the efficiency loss of the UE-CN mixed equilibrium.

\section{MIXED EQUILIBRIUM OF THE UE AND CN PLAYERS}

Let $G=(N, A)$ be a directed transportation network, where $N$ and $A$ denote the sets of nodes and links, respectively. $U$ represents the UE player (coveringall users following UE principle) in the network. Let $K$ be the set of $\mathrm{CN}$ players in the network; $W^{U}$ be the set of OriginDestination (OD) pairs where users obey UE principle; $W^{k}$ be the set of OD pairs where users are controlled by a CN player $k \in K$. Define $W^{K} \equiv \cup_{k \in K} W^{k}, W \equiv W^{U} \cup W^{K}$. Let $d_{w}$ be the demand between OD pair $w \in W ; R_{w}$ be the set of paths connecting OD pair $w \in W$.

Other notations used in this paper are as follows: $f_{r w}$ is the flow on path $r \in R_{w}, w \in W ; \delta_{a r}^{w}=1$ if the path $r \in R_{w}$ traverses link $a \in A$, and $\delta_{a r}^{w}=0$ otherwise; $v_{a}^{u}$ is the UE flow on link $a ; \mathbf{v}^{U} \equiv\left(\cdots, v_{a-1}^{U}, v_{a}^{U}, v_{a+1}^{U}, \cdots\right)$ is the vector of link flows by UE player; $v_{a}^{k}$ is the CN flow on link $a ; v_{a} \equiv\left(v_{a}^{U}, v_{a}^{1}, \cdots, v_{a}^{k}, \cdots\right)$ is the vector of all flows on link $a ; \mathbf{v}^{k} \equiv\left(\cdots, v_{a-1}^{k}, v_{a}^{k}, v_{a+1}^{k}, \cdots\right)$ is the vector of all link flows by $\mathrm{CN}$ player $k$;

$$
v_{a}^{K}=\sum_{k \in K} v_{a}^{k}
$$

is the total flow by all $\mathrm{CN}$ players on link $a$; $\mathbf{v}^{k} \equiv\left(\cdots, \mathbf{v}^{k-1}, \mathbf{v}^{k}, \mathbf{v}^{k+1}, \cdots\right) ; v_{a}=v_{a}^{u}+v_{a}^{k}$ is the total flow on link $a$. Define $\mathbf{v} \equiv\left(\mathbf{v}^{U}, \mathbf{v}^{K}\right)$. Let $t_{a}\left(v_{a}\right)$ denote the average cost of traversing link $a \in A$. This cost function is assumed to be separable in link flows and twice continuously differentiable.

Suppose all OD demands are fixed. Thus, the feasible sets of link flows by the UE player and the CN players can be defined as follows:

$$
\begin{aligned}
& \Omega^{U}=\left\{\begin{array}{l}
\mathbf{v}^{U} \mid v_{a}^{u}=\sum_{w \in W^{u}} \sum_{r \in R_{w}} f_{r w} \delta_{a r}^{w}, a \in A ; \\
\sum_{r \in R_{w}} f_{r w}=d_{w} ; f_{r w} \geq 0, r \in R_{w}, w \in W^{U}
\end{array}\right\} \\
& \Omega^{k}=\left\{\begin{array}{l}
\mathbf{v}^{k} \mid v_{a}^{k}=\sum_{w \in W^{k}} \sum_{r \in R_{w}} f_{r w} \delta_{a r}^{w}, a \in A ; \\
\sum_{r \in R_{w}} f_{r w}=d_{w} ; f_{r w} \geq 0, r \in R_{w}, w \in W^{k}
\end{array}\right\}, k \in K .
\end{aligned}
$$

All users in the UE player aim to minimize their personal travel costs under the current routing decisions of the $\mathrm{CN}$ players, which is equivalent to solve $\min _{v^{u} \in \Omega^{u}} \sum_{a \in A} \int_{0}^{v_{a}^{u}} t_{a}\left(v_{a}^{K}+x\right) d x$

where the variables $v_{a}^{K}, a \in A$ are taken as fixed. If $t_{a}\left(v_{a}\right)$ is strictly increasing, then the minimization problem (3) has a unique solution.

All users in the $\mathrm{CN}$ player $k \in K$ aim to minimize the total travel cost of this specific player under the current routing decision of other players, i.e.,

$\min _{v^{k} \in \Omega^{k}} \sum_{a \in A} t_{a}\left(v_{a}^{U}+v_{a}^{-k}+v_{a}^{k}\right) v_{a}^{k}$,

where

$$
v_{a}^{-k}=\sum_{i \in K, i \neq k} v_{a}^{i} .
$$

In (4), $v_{a}^{U}$ and $v_{a}^{-k}, a \in A, k \in K$ are taken as fixed, $v_{a}^{k}, k \in K$ as the variable. It is easy to show that if $t_{a}\left(v_{a}\right)$ is convex, the minimization problem (4) has a unique solution.

The solution simultaneously satisfying the optimality conditions of the minimization problems (3) and (4) is called the UE-CN mixed equilibrium solution. The condition for simultaneous minimization of the problems (3) and (4) can be formulated as the following variational inequity $(\mathrm{VI}):^{3}$

Lemma 1 Suppose $t_{a}\left(v_{a}\right), a \in A$ is strictly increasing and convex, the vector $\left(\mathbf{v}^{\text {mix, }}, \mathbf{v}^{\text {mix, } K}\right)$ is the UE-CN mixed equilibrium of (3) and (4) if and only if the following inequality holds

$\sum_{a \in A}\left\{t_{a}\left(v_{a}^{m i x}\right)\left(v_{a}^{u}-v_{a}^{m i x, u}\right)+\right.$

$\left.\sum_{k \in K}\left(t_{a}\left(v_{a}^{m i x, k}\right)+v_{a}^{m i x, k} t_{a}^{\prime}\left(v_{a}^{m i x}\right)\right)\left(v_{a}^{k}-V_{a}^{m i x, k}\right)\right\} \geq 0$,

$v^{u} \in \Omega^{u}, v^{k} \in \Omega^{k}, k \in K$.

where $v_{a}^{\text {mix }}=v_{a}^{\text {mix }, U}+v_{a}^{m i x, K}, a \in A$.

Since $t_{a}\left(V_{a}\right)$ is strictly increasing and convex, the VI problem (5) has at least one solution. Define the vector of the link costs perceived by the UE and $\mathrm{CN}$ players on link $a \in A$ as

$\mathbf{c}_{a}\left(\mathbf{v}_{a}\right)=\left(t_{a}\left(v_{a}\right), t_{a}\left(v_{a}\right)+v_{a}^{1} t_{a}^{\prime}\left(v_{a}\right)\right), \ldots, t_{a}\left(v_{a}\right)+v_{a}^{|K|} t_{a}^{\prime}\left(v_{a}\right)$,

where $\mathbf{v}_{a} \equiv\left(v_{a}^{U}, \mathbf{v}_{a}^{K}\right)$ and $|K|$ is the number of $\mathrm{CN}$ players. For arbitrary two flows $\mathbf{v}_{a} \neq \tilde{\mathbf{v}}_{a}$, if $\left\langle\left(c_{a}\left(\mathbf{v}_{a}\right)-c_{a}\left(\tilde{\mathbf{v}}_{a}\right)\right)^{T}, \mathbf{v}_{a}-\tilde{\mathbf{v}}_{a}\right\rangle>0$ holds, where $\langle\cdot, \cdot\rangle$ is inner product, then the $\mathrm{VI}$ problem (5) has at most one solution. ${ }^{13}$ It is obvious that the VI problem (5) has a unique solution if $t_{a}\left(v_{a}\right)$ is an affine and strictly monotone function for each link $a \in A$. When the link travel cost function is polynomial function, Boulogne et al. proved the uniqueness of the UE-CN mixed equilibrium. ${ }^{14}$ This is again described below.

Lemma 2 Suppose that the link travel cost function takes a polynomial form as $t_{a}\left(v_{a}\right)=t_{a 0}+\alpha_{a}\left(v_{a}\right)^{p}$, where $t_{a 0}$ is a nonnegative constant free flow travel cost on link $a \in A, \alpha_{a}$ is a link specific nonnegative parameter, and $0<p<p^{*}$. If the number of Nash players in problem (5) is limited, then the solution is unique. 
Here $p^{*}=(3|K|-1) /(K \mid-1)$ where $|K|$ is the number of $C N$ players in the network.

Let $\mathbf{v}^{\text {mix }}=\left(\mathbf{v}^{\text {mix }, U}, \mathbf{v}^{\text {mix, } K}\right)$ and $v_{A}^{\text {mix }}=\left(v_{a}^{\text {mix }}\right), a \in A$ be the solution vector of the $\mathrm{VI}$ problem (5) and the vector of the aggregate link flow in the UE-CN mixed equilibrium, respectively. Then, the total travel cost of the system can be written as

$$
\begin{gathered}
T\left(\mathbf{v}^{\text {mix }}\right)=\sum_{a \in A} t_{a}\left(v_{a}^{\text {mix }}\right) v_{a}^{\text {mix }}= \\
=\sum_{a \in A} t_{a}\left(v_{a}^{\text {mix }}\right) v_{a}^{\text {mix }, U}+\sum_{a \in A} \sum_{k \in K} t_{a}\left(v_{a}^{m i x}\right) v_{a}^{\text {mix }, k} .
\end{gathered}
$$

Let $\mathbf{v}^{\text {so }}=\left(\mathbf{v}^{\text {so, } U}, \mathbf{v}^{\text {so, } K}\right)$ and $\mathbf{v}_{A}^{\text {so }}=\left(v_{a}^{\text {so }}\right), a \in A$ respectively be the solution and the aggregate link flow of the following optimization problem:

$\min _{v \in \Omega} \sum_{a \in A} t_{a}\left(v_{a}\right) v_{a}$,

where

$$
\Omega=\Omega^{U} \times \prod_{k \in K} \Omega^{k} .
$$

If $t_{a}\left(v_{a}\right)$ is strictly increasing and convex, then the problem (7) has a unique solution in terms of the aggregate link flows $v_{a}$. We use $v_{a}^{\text {so }}$ to denote the unique link flow solution of the problem (7). Note that the link flows $v_{a}^{s o, U}$ and $v_{a}^{s o, k}, k \in K, a \in A$ may not be unique, but

$$
v_{a}^{s o}=v_{a}^{s o, U}+\sum_{k \in K} v_{a}^{s o, k}
$$

is unique. The efficiency loss of the UE-CN mixed equilibrium can be defined as follows:

$\rho=\frac{T\left(\mathbf{v}^{\text {mix }}\right)}{T\left(\mathbf{v}^{\text {so }}\right)}=\frac{\sum_{a \in A} t_{a}\left(v_{a}^{\text {mix }}\right) v_{a}^{\text {mix }}}{\sum_{a \in A} t_{a}\left(v_{a}^{\text {so }}\right) v_{a}^{s o}}$.

Since $T\left(\mathbf{v}^{\text {so }}\right)$ measures the minimal total travel cost of the system and $T\left(\mathbf{v}^{\text {mix }}\right)$ is the sum of all subsystems' minimal travel costs, hence $\rho \geq 1$ holds. Next we focus on finding the upper bound of $\rho$.

\section{BOUNDING THE EFFICIENCY LOSS OF UE-CN MIXED EQUILIBRIUM}

Let $\mathbf{v}^{\text {so }}=\left(\mathbf{v}^{\mathrm{so}, U}, \mathbf{v}^{\mathrm{so}, K}\right)$ be a solution of the minimization problem (7). Note that $\mathbf{v}^{s o, U} \in \Omega^{U}, \mathbf{v}^{\text {so,k }} \in \Omega^{k}$, $k \in K$. Substituting $v_{a}^{U}=v_{a}^{s o, U}$ and $v_{a}^{k}=v_{a}^{s o, k}$ in the $V I$ problem (5), for

$$
T\left(\mathbf{v}^{\text {mix }}\right)=\sum_{a \in A} t_{a}\left(v_{a}^{\text {mix }}\right) v_{a}^{\text {mix }}
$$

we can obtain the next chain of inequalities and equalities:

$$
\begin{aligned}
& T\left(v^{\text {mix }}\right) \leq \sum_{a \in A} t_{a}\left(v_{a}^{\text {mix }}\right) v_{a}^{\text {so, } U}+\sum_{a \in A} \sum_{k \in K} t_{a}\left(v_{a}^{\text {mix }}\right) v_{a}^{\text {so,k }}+ \\
& \quad+\sum_{a \in A} \sum_{k \in K} v_{a}^{\text {mix }, k} t_{a}^{\prime}\left(v_{a}^{\text {mix }}\right)\left(v_{a}^{\text {so,k }}-v_{a}^{\text {mix }, k}\right)= \\
& =\sum_{a \in A} t_{a}\left(v_{a}^{\text {mix }}\right) v_{a}^{\text {so }}+\sum_{a \in A} \sum_{k \in K} v_{a}^{\text {mix }, k} t_{a}^{\prime}\left(v_{a}^{\text {mix }}\right)\left(v_{a}^{\text {so,k }}-v_{a}^{\text {mix }, k}\right)= \\
& =\sum_{a \in A} t_{a}\left(v_{a}^{\text {so }}\right) v_{a}^{\text {so }}+\sum_{a \in A}\left(t_{a}\left(v_{a}^{\text {mix }}\right)-t_{a}\left(v_{a}^{\text {so }}\right)\right) v_{a}^{\text {so }}+
\end{aligned}
$$

$$
+\sum_{a \in A} \sum_{k \in K} v_{a}^{m i x, k} t_{a}^{\prime}\left(v_{a}^{m i x}\right)\left(v_{a}^{\text {so,k }}-v_{a}^{m i x, k}\right) .
$$

For

$$
v_{a}=v_{a}^{U}+\sum_{k \in K} v_{a}^{k},
$$

if $t_{a}\left(v_{a}\right)=t_{a 0}+\alpha_{a}\left(v_{a}\right)^{p}$, then $t_{a}^{\prime}\left(v_{a}\right)=\alpha_{a} p\left(v_{a}\right)^{p-1}$. It follows

$$
\begin{aligned}
0 & \leq T\left(\mathbf{v}^{\text {mix }}\right) \leq T\left(\mathbf{v}^{\mathrm{so}}\right)+\sum_{a \in A} \alpha_{a}\left(\left(v_{a}^{\text {mix }}\right)^{p}-\left(v_{a}^{\mathrm{so}}\right)^{p}\right) v_{a}^{\mathrm{so}}+ \\
& +\sum_{a \in A} \alpha_{a} p\left(v_{a}^{\text {mix }}\right)^{p-1} \sum_{k \in K} v_{a}^{\text {mix }, k}\left(v_{a}^{\mathrm{so}, k}-v_{a}^{\text {mix }, k}\right) .
\end{aligned}
$$

If we can find an upper bound for the sum of the second and the third terms in the right-hand side (RHS) of (9), we then obtain the efficiency loss of the VI problem (5). Now, we derive the upper bound of (8) by the scaling method and the nonlinear programming method, respectively. The scaling method is introduced as follows.

From inequality (9) we have

$$
\begin{aligned}
& T\left(\mathbf{v}^{\text {mix }}\right) \leq T\left(\mathbf{v}^{s o}\right)+\max _{v \in \Omega}\left\{\sum_{a \in A} \alpha_{a}\left(\left(v_{a}^{\text {mix }}\right)^{p}-\left(v_{a}\right)^{p}\right) v_{a}\right. \\
& \left.+\sum_{a \in A} \alpha_{a} p\left(v_{a}^{m i x}\right)^{p-1} \sum_{k \in K} v_{a}^{m i x, k}\left(v_{a}^{k}-v_{a}^{m i x, k}\right)\right\} \\
& \leq T\left(\mathbf{v}^{s o}\right)+\max _{v_{a}^{u} \geq 0, v_{a}^{k} \geq 0, k \in K, a \in A}\left\{\sum_{a \in A} \alpha_{a}\left(\left(v_{a}^{\text {mix }}\right)^{p}-\left(v_{a}\right)^{p}\right) v_{a}\right. \\
& \left.+\sum_{a \in A} \alpha_{a} p\left(v_{a}^{\text {mix }}\right)^{p-1} \sum_{k \in K} v_{a}^{\text {mix }, k}\left(v_{a}^{k}-v_{a}^{\text {mix }, k}\right)\right\} .
\end{aligned}
$$

Since the feasible region of $v_{a}^{U} \geq 0$ and $v_{a}^{k} \geq 0$, $k \in K$ for every link $a \in A$ is larger than that of $\Omega$, the last inequality of (10) is satisfied.

Consider the following inequality:

$$
\begin{gathered}
\sum_{k \in K} v_{a}^{m i x, k}\left(v_{a}^{k}-v_{a}^{m i x, k}\right) \leq \sum_{k \in K} \frac{1}{4}\left(v_{a}^{k}\right)^{2} \leq \\
\leq \frac{1}{4}\left(\sum_{k \in K} v_{a}^{k}\right)^{2} \leq \frac{1}{4}\left(v_{a}\right)^{2},
\end{gathered}
$$

where the first, second and last inequalities are satisfied respectively due to

$$
\begin{gathered}
\left(\frac{1}{2} v_{a}^{k}-v_{a}^{m i x, k}\right)^{2} \geq 0, \forall k \in K, v_{a}^{k} \geq 0 \text { and } \\
v_{a}=v_{a}^{u}+\sum_{k \in K} v_{a}^{k}, v_{a}^{u} \geq 0 .
\end{gathered}
$$

Since $\alpha_{a} p\left(v_{a}^{\text {mix }}\right)^{p-1} \geq 0$, inequality (10) is equivalent to $T\left(\mathbf{v}^{\text {mix }}\right) \leq T\left(\mathbf{v}^{\text {so }}\right)+\max _{v_{a} \geq 0}\left\{\sum_{a \in A} \alpha_{a}\left(\left(v_{a}^{\text {mix }}\right)^{p}-\left(v_{a}\right)^{p}\right) v_{a}+\right.$

$$
\left.+\sum_{a \in A} \alpha_{a} p\left(v_{a}^{m i x}\right)^{p-1} \frac{1}{4}\left(v_{a}\right)^{2}\right\}
$$

$$
\text { Let } F\left(v_{a}\right)=\left(v_{a}^{\text {mix }}\right)^{p} v_{a}+\frac{p}{4}\left(v_{a}^{\text {mix }}\right)^{p-1}\left(v_{a}\right)^{2}-\left(v_{a}\right)^{p+1} \text {, }
$$

$v_{a} \in[0,+\infty)$. Obviously, $F\left(v_{a}\right)$ is continuous in domain, so the second term in the RHS of (12) has the maximum within $v_{a} \in\left[0, v_{a}^{\text {mix }}\right]$ as long as we can obtain $F^{\prime}\left(v_{a}\right) \leq 0$ under the condition $v_{a} \geq v_{a}^{\text {mix }}$. It is easy to know

$$
F^{\prime}\left(v_{a}\right)=\left(v_{a}^{\text {mix }}\right)^{p}+\frac{p}{2}\left(v_{a}^{\text {mix }}\right)^{p-1} v_{a}-(p+1)\left(v_{a}\right)^{p},
$$




$$
\begin{gathered}
F^{\prime \prime}\left(v_{a}\right)=\frac{p}{2}\left(v_{a}^{\text {mix }}\right)^{p-1}-(p+1) p\left(v_{a}\right)^{p-1} \text { and } \\
F^{\prime \prime \prime}\left(v_{a}\right)=-(p+1) p(p-1)\left(v_{a}\right)^{p-2} .
\end{gathered}
$$

For $v_{a} \geq v_{a}^{\text {mix }} \geq 0$, we have $F^{\prime \prime \prime}\left(v_{a}\right) \leq 0$, this means $F^{\prime \prime \prime}\left(v_{a}\right)$ decreases with the variable $v_{a} \in\left[v_{a}^{\text {mix }},+\infty\right)$. Then we have

$$
\begin{aligned}
& F^{\prime \prime}\left(v_{a}\right) \leq F^{\prime \prime}\left(v_{a}^{\text {mix }}\right)=\frac{p}{2}\left(v_{a}^{\text {mix }}\right)^{p-1}-(p+1) p\left(v_{a}^{\text {mix }}\right)^{p-1}= \\
& =-\left(p^{2}+p / 2\right)\left(v_{a}^{\text {mix }}\right)^{p-1} \leq 0 .
\end{aligned}
$$

The inequality (13) shows that $F^{\prime}\left(v_{a}\right)$ decreases with the variable $v_{a} \in\left[v_{a}^{\text {mix }},+\infty\right)$. Since

$$
F^{\prime}\left(v_{a}^{\text {mix }}\right)=-\frac{p}{2}\left(v_{a}^{\text {mix }}\right)^{p} \leq 0,
$$

we get $F^{\prime}\left(v_{a}\right) \leq F^{\prime}\left(v_{a}^{\text {mix }}\right) \leq 0$ under the condition $v_{a} \geq v_{a}^{\text {mix }}$. Thus, we conclude that the second term in the RHS of (12) has the maximum within $\left[0, v_{a}^{m i x}\right]$. Rewrite the inequality (12) as

$$
\begin{gathered}
T\left(\mathbf{v}^{\text {mix }}\right) \leq T\left(\mathbf{v}^{\text {so }}\right)+\max _{v_{a} \geq 0}\left\{\sum_{a \in A} \alpha_{a}\left(\left(v_{a}^{\text {mix }}\right)^{p}-\left(v_{a}\right)^{p}\right) v_{a}+\right. \\
\left.+\sum_{a \in A} \alpha_{a} p\left(v_{a}^{\text {mix }}\right)^{p-1} \frac{1}{4}\left(v_{a}\right)^{2}\right\}= \\
=T\left(\mathbf{v}^{\text {so }}\right)+\max _{u \in[0,1]}\left\{\sum_{a \in A} \alpha_{a}\left(\left(v_{a}^{\text {mix }}\right)^{p}-u^{p}\left(v_{a}^{\text {mix }}\right)^{p}\right) u v_{a}^{\text {mix }}+\right. \\
\left.+\sum_{a \in A} \alpha_{a} p\left(v_{a}^{\text {mix }}\right)^{p-1} \frac{1}{4}\left(u v_{a}^{\text {mix }}\right)^{2}\right\}= \\
=T\left(\mathbf{v}^{\text {so }}\right)+\max _{u \in[0,1]}\left(u+\frac{p}{4} u^{2}-u^{p+1}\right) \sum_{a \in A} \alpha_{a}\left(v_{a}^{\text {mix }}\right)^{p+1} \leq \\
\leq T\left(\mathbf{v}^{\text {so }}\right)+\max _{u \in[0,1]}\left(u+\frac{p}{4} u^{2}-u^{p+1}\right) \\
\sum_{a \in A}\left(\alpha_{a}\left(v_{a}^{\text {mix }}\right)^{p}+t_{a 0}\right) v_{a}^{\text {mix }}= \\
\leq T\left(\mathbf{v}^{\text {so }}\right)+\max _{u \in[0,1]}\left(u+\frac{p}{4} u^{2}-u^{p+1}\right) T\left(v^{\text {mix }}\right) .
\end{gathered}
$$

The first equation is satisfied as $v_{a}=u v_{a}^{\text {mix }}$, $u \in[0,1]$. Because of (8), $\rho \geq 1$, so we have to define

$$
\left(1-\max _{u \in[0,1]}\left(u+\frac{p}{4} u^{2}-u^{p+1}\right)\right)^{-1} \equiv+\infty
$$

in the case

$$
\max _{u \in[0,1]}\left(u+\frac{p}{4} u^{2}-u^{p+1}\right) \geq 1 .
$$

Thus we have the following theorem:

Theorem 1 Suppose that link travel cost function $t_{a}\left(v_{a}\right)$, $a \in A$ is a polynomial function and $p$ is the highest degree of the polynomial function, $\mathbf{v}^{\text {mix }}=\left(\mathbf{v}^{\text {mix }, U}, \mathbf{v}^{\text {mix }, K}\right)$, $\mathbf{v}^{\text {mix, } U} \in \Omega^{U}, \mathbf{v}^{\text {mix,k }} \in \Omega^{k}, k \in K$ is a solution of problem (5) and $\mathbf{v}^{\text {so }}=\left(\mathbf{v}^{\text {so, } U}, \mathbf{v}^{\text {so,K }}\right), \mathbf{v}^{\text {so, } U} \in \Omega^{U}, \mathbf{v}^{\text {so,k }} \in \Omega^{k}, k \in K$ is a solution of problem (7). Then the efficiency loss $\rho$ of the UE-CN mixed equilibrium satisfies

$\rho \leq\left(1-\max _{u \in[0,1]}\left(u+\frac{p}{4} u^{2}-u^{p+1}\right)\right)^{-1}$.

Corollary 1. If all link travel cost functions are affine functions, then the efficiency loss of the VI problem (5) is not greater than $3 / 2$.

Proof: Let $p=1$ in Theorem 1, then

$$
\begin{aligned}
\max _{u \in[0,1]}\left(u+\frac{p}{4} u^{2}-u^{p+1}\right) & =\max _{u \in[0,1]}\left(u-\frac{3}{4} u^{2}\right)=\frac{1}{3} \\
\text { when } u & =\frac{2}{3} .
\end{aligned}
$$

From (15), we have

$$
\rho \leq \frac{3}{2} .
$$

The above analyses show that in the scaling method, the efficiency loss of the UE-CN mixed equilibrium is irrelevant to the number of $\mathrm{CN}$ players in the network.

Next, we use the nonlinear programming method to further discuss the efficiency loss. In order to get the upper bound for the sum of the second and third terms in the RHS of (9), we first investigate the following nonlinear programming:

$$
\begin{aligned}
\max _{v_{a} \geq 0}\{ & \alpha_{a}\left(\left(v_{a}^{\text {mix }}\right)^{p}-\left(v_{a}\right)^{p}\right) v_{a}+ \\
& \left.+\alpha_{a} p\left(v_{a}^{\text {mix }}\right)^{p-1} \sum_{k \in K} v_{a}^{\text {mix }, k}\left(v_{a}^{k}-v_{a}^{m i x}, k\right)\right\}, a \in A .
\end{aligned}
$$

Let

$$
\begin{aligned}
G\left(v_{a}\right) & =\alpha_{a}\left(\left(v_{a}^{m i x}\right)^{p}-\left(v_{a}\right)^{p}\right) v_{a}+ \\
& +\alpha_{a} p\left(v_{a}^{m i x}\right)^{p-1} \sum_{k \in K} v_{a}^{m i x, k}\left(v_{a}^{k}-v_{a}^{m i x, k}\right) .
\end{aligned}
$$

Since the Hessian matrix of $G\left(\mathbf{v}_{a}\right)$ is negative semidefinite matrix, function $G\left(\mathbf{v}_{a}\right)$ is a concave function of the variables $v_{a}^{U} \geq 0$ and $v_{a}^{k} \geq 0, k \in K$ in the case $p>0$. Then we conclude $G\left(\mathbf{v}_{a}\right)$ has a global maximum. Let $\lambda_{a}^{U}$ and $\lambda_{a}^{k}, a \in A$ be the Lagrange multipliers associated with $v_{a}^{U} \geq 0$ and $v_{a}^{k} \geq 0, k \in K$. Then we get the first-order optimality conditions of (16) as follows:

$$
\begin{aligned}
& \alpha_{a}\left(v_{a}^{\text {mix }}\right)^{p}-\alpha_{a}(p+1)\left(v_{a}\right)^{p}+\lambda_{a}^{U}=0, \\
& \lambda_{a}^{U} \geq 0, \lambda_{a}^{U} v_{a}^{U}=0, \\
& \alpha_{a}\left(v_{a}^{\text {mix }}\right)^{p}-\alpha_{a}(p+1)\left(v_{a}\right)^{p}+ \\
& \quad \quad+\alpha_{a} p\left(v_{a}^{\text {mix }}\right)^{p-1} v_{a}^{\text {mix }, k}+\lambda_{a}^{k}=0, k \in K, \\
& \lambda_{a}^{k} \geq 0, \lambda_{a}^{k} v_{a}^{k}=0, k \in K .
\end{aligned}
$$

Combining (17) with (18), we have

$$
v_{a} \geq\left(\frac{1}{p+1}\right)^{1 / p} v_{a}^{\text {mix }} \text {. }
$$

For each given link, we can find that problem (16) reaches the maximum with one and only one of the following two conditions:

$$
\begin{aligned}
& v_{a}=\left(\frac{1}{p+1}\right)^{1 / p} v_{a}^{\text {mix }}, \\
& v_{a}>\left(\frac{1}{p+1}\right)^{1 / p} v_{a}^{\text {mix }} .
\end{aligned}
$$

If condition (21) holds, it is easy to get $\lambda_{a}^{U}=0$ and $v_{a}^{U} \geq 0$ from (17) and (18). Substituting (21) into (19), we obtain

$\alpha_{a} p\left(v_{a}^{m i x}\right)^{p-1} v_{a}^{m i x, k}+\lambda_{a}^{k}=0, k \in K$,

which are fulfilled only for $v_{a}^{m i x, k}=0$ and $\lambda_{a}^{k}=0, k \in K$, according to (20). In that occasion, we have

$\alpha_{a}\left(\left(v_{a}^{m i x}\right)^{p}-\left(v_{a}\right)^{p}\right) v_{a}+\alpha_{a} p\left(v_{a}^{m i x}\right)^{p-1} \sum_{k \in K} v_{a}^{m i x, k}\left(v_{a}^{k}-v_{a}^{m i x, k}\right)$ 


$$
\begin{aligned}
& =\alpha_{a}\left(\left(v_{a}^{\text {mix }}\right)^{p}-\left(\left(\frac{1}{p+1}\right)^{1 / p} v_{a}^{\text {mix }}\right)^{p}\right)\left(\frac{1}{p+1}\right)^{1 / p} v_{a}^{\text {mix }} \\
& =\alpha_{a}\left(\frac{1}{1+p}\right)\left(\frac{1}{1+p}\right)^{1 / p}\left(v_{a}^{\text {mix }}\right)^{p+1} \\
& \leq\left(\frac{p}{1+p}\right)\left(\frac{1}{1+p}\right)^{1 / p} t_{a}\left(v_{a}^{\text {mix }}\right) v_{a}^{\text {mix }} .
\end{aligned}
$$

The last inequality of (24) follows from the fact that $t_{a 0} \geq 0, a \in A$.

If condition (22) holds, referring to (17) and (18), we have $\lambda_{a}^{U}>0$ and $v_{a}^{U}=0$. Next, we study the relation between the efficiency loss and the set $K$ through analyzing the following two cases.

Case 1: $|K|=1$.

In this case, $v_{a}=v_{a}^{c n}$ where $v_{a}^{c n}$ denotes the flow of CN player on link $a$ and $V_{a}^{\text {mix,cn }}=\kappa_{a} v_{a}^{\text {mix }}, 0 \leq \kappa_{a} \leq 1$ where by convention $\kappa_{a}=1$ if $v_{a}^{\text {mix,cn }}=v_{a}^{\text {mix }}=0$. Substituting $v_{a}^{m i x, k}=v_{a}^{m i x, c n}$ and $v_{a}=v_{a}^{c n}$ into (19), we get

$$
v_{a}=v_{a}^{c n}=\left(\frac{1+p \kappa_{a}}{1+p}\right)^{1 / p} v_{a}^{\text {mix }} \text {. }
$$

From (16), we have

$$
\begin{aligned}
& \max _{v_{a} \geq 0}\{ \alpha_{a}\left(\left(v_{a}^{\text {mix }}\right)^{p}-\left(v_{a}\right)^{p}\right) v_{a}+ \\
&\left.+\alpha_{a} p\left(v_{a}^{\text {mix }}\right)^{p-1} v_{a}^{\text {mix }, c n}\left(v_{a}^{c n}-v_{a}^{\text {mix }, c n}\right)\right\}= \\
&=\alpha_{a}\left(1-\kappa_{a}\right)\left(\frac{p}{1+p}\right)\left(\frac{1+p \kappa_{a}}{1+p}\right)^{1 / p}\left(v_{a}^{\text {mix }}\right)^{p+1}+ \\
& \quad+\alpha_{a} p\left[\left(\frac{1+p \kappa_{a}}{1+p}\right)^{1 / p}-\kappa_{a}\right] \kappa_{a}\left(v_{a}^{\text {mix }}\right)^{p+1}= \\
&=\alpha_{a} \eta_{a}\left(v_{a}^{\text {mix }}\right)^{p+1} \leq \\
& \leq \eta_{a} t_{a}\left(v_{a}^{\text {mix }}\right) v_{a}^{\text {mix }},
\end{aligned}
$$

where

$$
\begin{aligned}
\eta_{a}= & \left(1-\kappa_{a}\right)\left(\frac{p}{1+p}\right)\left(\frac{1+p \kappa_{a}}{1+p}\right)^{1 / p}+ \\
& +p\left[\left(\frac{1+p \kappa_{a}}{1+p}\right)^{1 / p}-\kappa_{a}\right] \kappa_{a} .
\end{aligned}
$$

The inequality of (25) is satisfied from the fact that $t_{a 0} \geq 0, a \in A$.

Case 2: $|K| \geq 2$.

When $v_{a}^{m i x, k_{1}}>v_{a}^{m i x, k_{2}}$, from (19) and (20) we can get $\lambda_{a}^{k_{1}}<\lambda_{a}^{k_{2}}$ and $v_{a}^{k_{1}} \geq v_{a}^{k_{2}}=0$. Let $v_{a}^{m i x, \bar{k}}=\max _{k \in K}\left(v_{a}^{m i x, k}\right)$ and

$$
\beta_{a}=\frac{v_{a}^{\text {mix }, \bar{k}}}{v_{a}^{\text {mix }}}, \gamma_{a}=\frac{v_{a}^{\text {mix }, U}}{v_{a}^{\text {mix }}}
$$

for $a \in A$ with $0 \leq \beta_{a} \leq 1,0 \leq \gamma_{a} \leq 1,0 \leq \beta_{a}, \gamma_{a} \leq 1$. From (19), it is easy to get $v_{a}^{\bar{k}}>0$ and $v_{a}^{k}=0$ for $k \neq \bar{k}$. Since

$$
v_{a}^{U}=0 \text { and } v_{a}=v_{a}^{U}+\sum_{k \in K} v_{a}^{k},
$$

we have

$v_{a}=v_{a}^{\bar{k}}=\left(\frac{p \beta_{a}+1}{1+p}\right)^{1 / p} v_{a}^{\text {mix }}, a \in A$

Therefore, we can obtain the followivng result from (16):

$\max _{v_{a} \geq 0}\left\{\alpha_{a}\left(\left(v_{a}^{\operatorname{mix}}\right)^{p}-\left(v_{a}\right)^{p}\right) v_{a}+\right.$

$$
\begin{aligned}
& \left.+\alpha_{a} p\left(v_{a}^{\text {mix }}\right)^{p-1} \sum_{k \in K} v_{a}^{\text {mix }, k}\left(v_{a}^{k}-v_{a}^{\text {mix }, k}\right)\right\}= \\
& =\alpha_{a}\left(1-\beta_{a}\right)\left(\frac{p}{1+p}\right)\left(\frac{1+p \beta_{a}}{1+p}\right)^{1 / p}\left(v_{a}^{\text {mix }}\right)^{p+1}+ \\
& +\alpha_{a} p\left[\left(\frac{1+p \beta_{a}}{1+p}\right)^{1 / p}-\beta_{a}\right] \beta_{a}\left(v_{a}^{\text {mix }}\right)^{p+1}= \\
& \quad-\alpha_{a} p\left(v_{a}^{\text {mix }}\right)^{p-1} \sum_{k \in K, k \neq \bar{k}}\left(v_{a}^{\text {mix }, k}\right)^{2} \leq \\
& \leq \alpha_{a} S_{a}\left(v_{a}^{\text {mix }}\right)^{p+1} \leq, \\
& \leq S_{a} t_{a}\left(v_{a}^{\text {mix }}\right) v_{a}^{\text {mix }},
\end{aligned}
$$

where

$S_{a}=\left(1-\beta_{a}\right)\left(\frac{p}{1+p}\right)\left(\frac{1+p \beta_{a}}{1+p}\right)^{1 / p}+$

$+p\left[\left(\frac{1+p \beta_{a}}{1+p}\right)^{1 / p}-\beta_{a}\right] \beta_{a}-p \frac{\left(1-\beta_{a}-\gamma_{a}\right)^{2}}{|K|-1}$.

The first and second inequalities of (28) follow from the fact that

$$
\begin{aligned}
& \sum_{k \in K, k \neq \bar{k}}\left(v_{a}^{m i x, k}\right)^{2} \geq \frac{1}{|K|-1}\left(\sum_{k \in K, k \neq \bar{k}} v_{m}^{\text {mix }, k}\right)^{2}= \\
= & \frac{\left(1-\beta_{a}-\gamma_{a}\right)}{|K|-1}\left(v_{a}^{\text {mix }}\right)^{2} \text { and } t_{a 0} \geq 0, a \in A .
\end{aligned}
$$

In this paper, we mainly consider the network with both UE and CN players. If $|K|=1$, (24) or (25) will be satisfied when (16) gets the maximum. Let

$\psi_{a}=\max \left\{\left(\frac{p}{1+p}\right)\left(\frac{1}{1+p}\right)^{1 / p}, \eta_{a}\right\}$,

and

$\psi=\max _{a \in A} \psi_{a}$.

Thus, inequality (9) can be rewritten as

$T\left(\mathbf{v}^{\text {mix }}\right) \leq T\left(\mathbf{v}^{\text {so }}\right)+\psi T\left(\mathbf{v}^{\text {mix }}\right)$.

If $|K| \geq 2$, (24) or (28) is satisfied when (16) has the maximum. Let

$\xi_{a}=\max \left\{\left(\frac{p}{1+p}\right)\left(\frac{1}{1+p}\right)^{1 / p}, S_{a}\right\}$

and

$\xi=\max _{a \in A} \xi_{a}$.

Then, inequality (9) can be rewritten as

$T\left(\mathbf{v}^{\text {mix }}\right) \leq T\left(\mathbf{v}^{\text {so }}\right)+\xi T\left(\mathbf{v}^{\text {mix }}\right)$.

Finally, we get the following theorem:

Theorem 2 Suppose the link travel cost function $t_{a}\left(v_{a}\right)$, $a \in A$ is a polynomial function, $\mathbf{v}^{\text {mix }}=\left(\mathbf{v}^{\text {mix }, U}, \mathbf{v}^{\text {mix }, K}\right)$, $\mathbf{v}^{\text {mix }, U} \in \Omega^{U}, \mathbf{v}^{\text {mix,k }} \in \Omega^{k}, k \in K$ be a solution of the VI problem (5) and $\mathbf{v}^{\text {so }}=\left(\mathbf{v}^{s o, U}, \mathbf{v}^{s o, K}\right), \mathbf{v}^{\text {so, } U} \in \Omega^{U}$, $\mathbf{v}^{\text {so,k }} \in \Omega^{k}, k \in K$ be a solution of problem (7). We have

(1) if $|K|=1$, then $\rho \leq \frac{1}{1-\psi}$;

(2) if $|K| \geq 2$, then $\rho \leq \frac{1}{1-\xi}$.

where $K$ is the set of $\mathrm{CN}$ players in the network, $|K|$ is the number of $\mathrm{CN}$ players, $\psi$ is defined by equations (26), (30) and (31), $\xi$ by equations (29), (33) and (34). 


\section{A NUMERICAL EXAMPLE}

Consider a directed graph consisting of 4 nodes and 5 links (see Figure 1). The link travel cost functions are defined as $t_{1}=1.8, t_{2}=0, t_{3}=v_{3}, t_{4}=0$, $t_{5}=2.6$, respectively. There are two OD pairs, namely $(1,4)$ and $(2,4)$ with fixed demands $d_{14}=1$ and $d_{24}=1$, respectively.

The SO link flow solution can be obtained by solving the following minimization problem:

$$
\min 1.8 v_{1}+\left(v_{3}\right)^{2}+2.6 v_{5}
$$

$$
\begin{array}{ll}
\text { s.t. } & v_{1}+v_{2}=1 \\
& v_{2}+v_{4}=v_{3} \\
& v_{4}+v_{5}=1 \\
& v_{i} \geq 0, i=1, \ldots, 5 .
\end{array}
$$

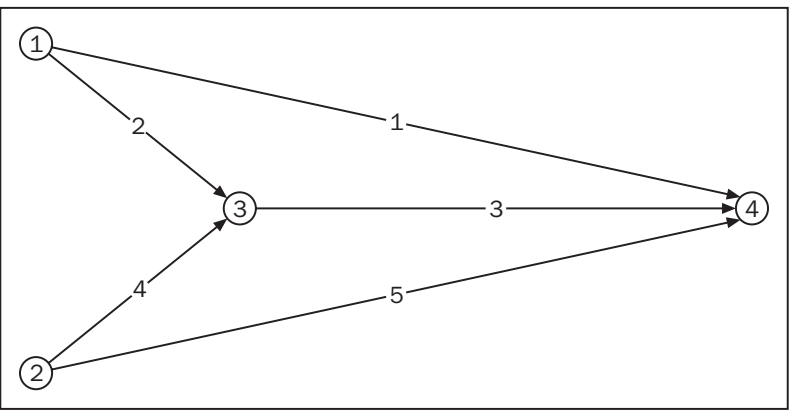

Figure 1 - The network used in the example.

The optimal solution is $v_{1}^{\text {so }}=1, \quad v_{2}^{\text {so }}=0$, $v_{3}^{\text {so }}=v_{4}^{\text {so }}=1, v_{5}^{v o}=0$ and the total travel cost is 2.8 .

Suppose OD pair $(1,4)$ is governed by users belonging to UE player and OD pair $(2,4)$ by the $C N$ player. The UE-CN mixed equilibrium is obtained by solving the following two minimization problems simultaneously:

$$
\min z_{1}=\int_{0}^{v_{1}^{u}} 1.8 \mathrm{~d} x+\int_{0}^{v_{2}^{u}} 0 \mathrm{~d} x+\int_{0}^{v_{3}^{u}} 1.8\left(v_{3}^{1}+x\right) \mathrm{d} x
$$

s.t. $\quad v_{1}^{U}+v_{2}^{U}=1$

$$
\begin{aligned}
& v_{2}^{U}=v_{3}^{U} \\
& v_{i}^{U} \geq 0, i=1,2,3 .
\end{aligned}
$$

and

$$
\min z_{2}=2.6 v_{5}^{1}+0 v_{4}^{1}+\left(v_{3}^{U}+v_{3}^{1}\right) v_{3}^{1}
$$

s.t. $\quad v_{4}^{1}+v_{5}^{1}=1$

$v_{4}^{1}=v_{3}^{1}$

$$
v_{3}^{1}, v_{4}^{1}, v_{5}^{1} \geq 0 \text {. }
$$

i.e.,

and

$$
\min _{0 \leq v_{3}^{U} \leq 1} z_{1}=1.8-1.8 v_{3}^{U}+v_{3}^{1} v_{3}^{U}+\frac{1}{2}\left(v_{3}^{U}\right)^{2}
$$

$$
\min _{0 \leq v_{3}^{1} \leq 1} z_{2}=2.6-2.6 v_{3}^{1}+v_{3}^{1} v_{3}^{U}+\left(v_{3}^{1}\right)^{2}
$$

The necessary condition

$$
\frac{d z_{1}}{d v_{3}^{U}}=0
$$

for reaching the first minimum gives $v_{3}^{1}+v_{3}^{U}-1.8=0$ and the necessary condition

$$
\frac{d z_{2}}{d v_{3}^{1}}=0
$$

for realizing the second minimum leads to $2 v_{3}^{1}+v_{3}^{U}-2.6=0$. The UE-CN mixed equilibrium solution is as follows:

$$
\begin{gathered}
v_{1}^{m i x, c n}=0, v_{2}^{m i x, c n}=0, v_{3}^{m i x, c n}=0.8, v_{4}^{m i x, c n}=0.8, \\
v_{5}^{m i x, c n}=0.2, v_{1}^{m i x, U}=0, v_{2}^{m i x, U}=1, v_{3}^{\text {mix }, U}=1, \\
v_{4}^{m i x, U}=0, v_{5}^{m i x, U}=0 .
\end{gathered}
$$

The aggregate link flow is $v_{1}^{\text {mix }}=0, v_{2}^{\text {mix }}=1$, $v_{3}^{\text {mix }}=1.8, v_{4}^{\text {mix }}=0.8, v_{5}^{\text {mix }}=0.2$ which generates the system's total travel cost 3.76. Thus, the efficiency loss is $\rho=3.76 / 2.8=1.3429$.

Result $\rho=1.3429 \leq 1.5$ satisfies the statement in Corollary 1. Based on the definition $v_{a}^{\text {mix }, c n}=\kappa_{a} v_{a}^{\text {mix }}$, $0 \leq \kappa_{a} \leq 1 \quad$ where by convention $\kappa_{a}=1$ if $v_{a}^{m i x, c n}=v_{a}^{m i x}=0$. Then, $\kappa_{1}=1, \kappa_{2}=0, \kappa_{3}=0.4444$, $\kappa_{4}=\kappa_{5}=1$. Considering $p=1$ and

$$
\begin{aligned}
\eta_{a}= & \left(1-\kappa_{a}\right)\left(\frac{p}{1+p}\right)\left(\frac{1+p \kappa_{a}}{1+p}\right)^{1 / p}+ \\
& +p\left[\left(\frac{1+p \kappa_{a}}{1+p}\right)^{1 / p}-\kappa_{a}\right] \kappa_{a},
\end{aligned}
$$

we get $\eta_{1}=0, \eta_{2}=0, \eta_{3}=0.3241, \eta_{4}=\eta_{5}=0$. For

$$
\psi_{a}=\max \left\{\left(\frac{p}{1+p}\right)\left(\frac{1}{1+p}\right)^{1 / p}, \eta_{a}\right\}
$$

and

we obtain

$$
\psi=\max _{a \in A} \psi_{a}
$$

$$
\begin{gathered}
\psi_{1}=\psi_{2}=0.25, \psi_{3}=0.3241 \\
\psi_{4}=\psi_{5}=0.25 \text { and } \psi=0.3241
\end{gathered}
$$

Thus, the bound becomes $\rho \leq(1-\psi)^{-1}=1.4795$, according to Theorem 2 . It should be noted that the computational burden of the scaling method is less than the nonlinear programming method, but the upper bound of efficiency loss given by the nonlinear programming method is more tight than that by the scaling method

\section{CONCLUSIONS}

In this paper, we have investigated the upper bounds of the efficiency loss in a network with both UE player and $\mathrm{CN}$ players, respectively, using the scaling method and the nonlinear programming method. The analytical results show that the upper bound obtained by each of the two methods depends on the polynomial degree of the link travel cost functions. Furthermore, the upper bound by the nonlinear programming method is relevant to the number of $\mathrm{CN}$ players. The numerical results illustrate that our analytical results are reasonable and the scaling method is easier to 
implement than the nonlinear programming method, but gives a larger upper bound. This states that for getting a smaller upper bound of the efficiency loss, more factors should be taken into account. Our ongoing study is to explore the efficiency loss of the UE-CN mixed equilibrium with elastic demand.

\section{Acknowledgement}

This research was partly supported by the National Natural Science Foundation of China (70821061, 70931160447) and the National Basic Research Program of China (2006CB705503).

\section{摘要}

\section{多项式路段成本函数下混合均衡行为的效率损失}

研究了同时存在用户均衡 (UE) 局中人和Cournot$\operatorname{Nash}(\mathrm{CN})$ 局中人时自私路径选择导致的效率损失问题。运 用放缩法和非线性规划方法分别得到了 UE-CN混合均衡在 多项式成本函数下的效率损失上界. 研究结果表明: 运用 放缩法得到的效率损失上界值依赖于路段多项式成本函数 对应的最高次; 运用非线性规划方法得到的效率损失上界 值不仅和路段多项式函数的最高次有关，而且还和 $\mathrm{CN}$ 局中 人的数目相关. 数值试验验证了得到的解析结论.

\section{关键词}

效率损失, UE-CN混合均衡, 变分不等式, 路径博弈

\section{REFERENCES}

[1] Wardrop, J. G.: Some theoretical aspects on road traffic research, Proceedings of the Institution of Civil Engineers, 1952

[2] Haurie, A., Marcotte, P.: On the relationship between Nash-Cournot and Wardrop equilibria, Networks, Vol.15, No. 3, 1985, pp. 295-308
[3] Harker, P. T.: Multiple equilibrium behaviors on networks, Transportation Science, Vol. 22, No. 1, 1988, pp. $39-46$

[4] Yang, H., Zhang, X. N., Meng, Q.: Stackelberg games and multiple equilibrium behaviors on networks, Transportation Research Part B, Vol. 41, No. 8, 2007, pp. 841-861

[5] Yang, H., Zhang, X. N.: Existence of anonymous link tolls for system optimum on networks with mixed equilibrium behaviors, Transportation Research Part B, Vol. 42, No .2, 2008, pp. 99-112

[6] Papadimitriou, C. H.: Algorithms, games, and the internet, Proceedings of the $33^{\text {rd }}$ Annual ACM Symposium on the theory of computing, Hersonissos, Greece, 2001

[7] Roughgarden, T., Tardos, E.: How bad is selfish routing? Journal of the ACM, Vol. 49, No. 2, 2002, pp. 236259

[8] Roughgarden, T., Tardos, E.: The price of anarchy is independent of the network topology, Journal of Computer and System Science, Vol. 67, No. 2, 2003, pp. 341-364

[9] Roughgarden, T.: Selfish Routing and the Price of Anarchy, The MIT Press, Cambridge, 2005

[10] Yang, H., Huang, H. J.: Mathematical and Economic Theory of Road Pricing, Elsevier Ltd, Oxford, UK, 2005

[11] Cominetti, R., Correa, J.R., Stier-Moses, N.E.: Network games with atomic players. Proceedings of the $33^{\text {rd }} \mathrm{An}$ nual International Colloquium in Automata, Languages, and Programming (ICALP), Spring-Verlag, Berlin Heidelberg, Germany, 2006

[12] Yang, H., Han, D.R., Lo, H. K.: Efficiency of atomic splittable selfish routing with polynomial cost functions, Network and Spatial Economics, Vol. 8, No. 4, 2008, pp. 443-451

[13] Kinderlehrer, D., Stampacchia, G.: An Introduction to Variational Inequalities and Their Applications, Academic Press, New York, 1986

[14] Boulogne, T., Altman, E., Kameda, H., Pourtallier, O.: Mixed equilibrium for multi-class routing games, IEEE Transactions on Automatic Control, Vol.47, No. 6, 2002, pp. 903-916 